\title{
Road Sign Analysis Using Multisensory Data
}

\author{
R.J. López-Sastre, S. Lafuente-Arroyo, P. Gil-Jiménez, P. Siegmann, \\ and S. Maldonado-Bascón \\ University of Alcalá, Department of Signal Theory and Communications \\ Polytechnic School, A-2 Km. 33,600 - 28805 - Alcalá de Henares - Madrid, Spain \\ \{robertoj.lopez, sergio.lafuente, pedro.gil, philip.siegmann, \\ saturnino.maldonado\}@uah.es
}

\begin{abstract}
This paper deals with the problem of estimating the following road sign parameters: height, dimensions, visibility distance and partial occlusions. This work belongs to a framework whose main applications involve road sign maintenance, driver assistance, and inventory systems. From this paper we suggest a multisensory system composed from two cameras, a GPS receiver, and a distance measurement device, all of them installed in a car. The process consists of several steps which include road sign detection, recognition and tracking, and road signs parameters estimation. From some trigonometric properties, and a camera model, the information provided by the tracking subsystem and the distance measurement sensors, we estimate the road signs parameters. Results show that the described calculation methodology offers a correct estimation for all types of traffic signs.
\end{abstract}

\section{Introduction}

Several works have recently focused on traffic sign detection and recognition [1, [2], 3], 4], 5] and [6. Specifically, in [7] it is described the framework that we use in this research. In this paper we handle the task of automatically estimating height, dimensions, visibility distance and partial occlusions of road signs. The aim of this work is to get an efficient framework which could be used in a inventory system, which could provide all of these estimated parameters for each sign, and not only the type. From this work, we present a complete development based on trigonometric relationships and a camera model to estimate the listed before parameters of road signs.

This paper is organized as follows. Section II shows a global vision of our system, and in section III we describe all the implemented algorithms to achieve the traffic sign parameters estimation. Sections IV and V report the obtained results and present the conclusions of this work, respectively.

\section{System Overview}

For this work we have built a complete inventory system as it is shown in Fig. 1. which has the following subsystems:

J. Mira and J.R. Álvarez (Eds.): IWINAC 2007, Part II, LNCS 4528, pp. 251-260 2007.

(C) Springer-Verlag Berlin Heidelberg 2007 
- Capture subsystem. We have mounted two firewire cameras on a car. The first one is entrusted to capture the road signs with a framerate of 15 fps (frames per second), and with the second one we want to capture the milestones of the route. This subsystem synchronizes the capture of these two cameras, and it saves the captured images in the hard disk of a laptop.

- A Positioning subsystem which is composed of a GPS (Global Positioning System) receiver and a Distance Measurement Device (DMD). The aim of this block is to acquire the GPS position of the vehicle, each second, and to measure the covered distance by the car. This subsystem sends all of these measured data to the Traffic sign analysis subsystem.

- Traffic sign analysis subsystem, which will develop two task. Firstly, the systems tries to detect, recognize and track the traffic signs in the images, and in a second step, the system performs the estimation of parameters.

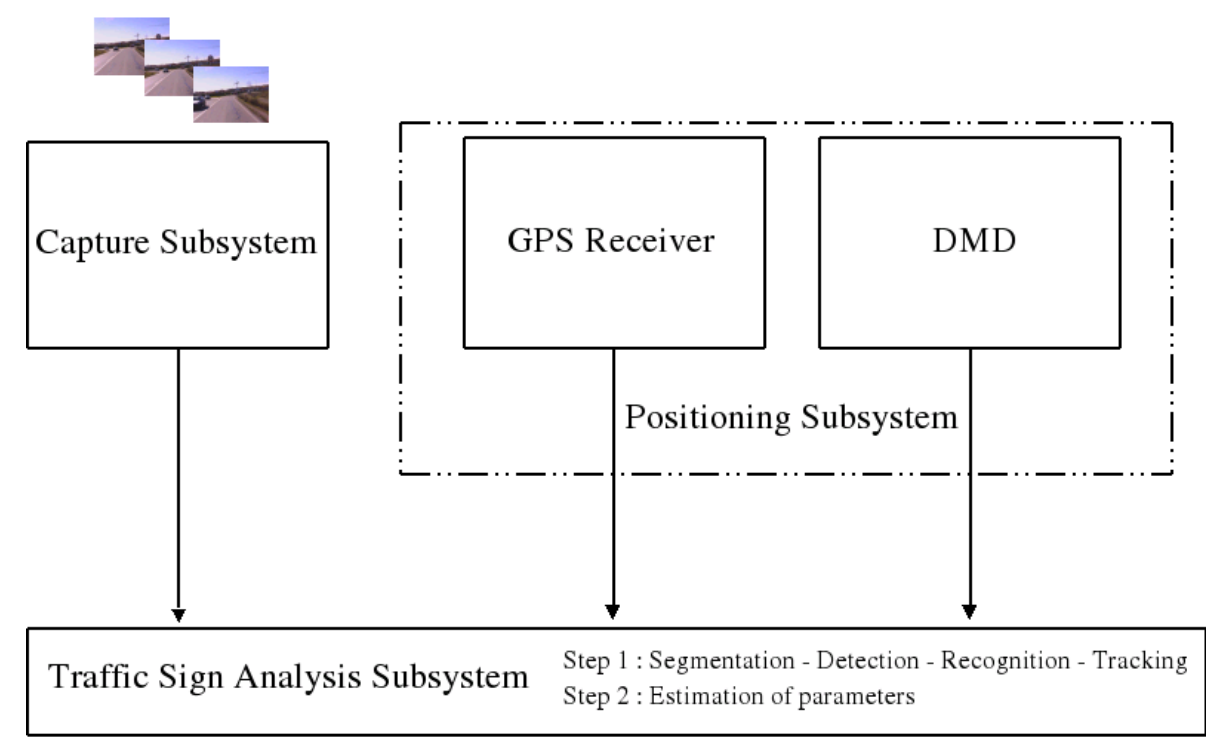

Fig. 1. Diagram with all subsystems which are part of the complete system

In Fig. 2 we show a complete flow diagram with all the tasks that this system realizes. In the last step it is where we implement the parameters estimation process which is the main focus of this paper. It is important to note that this estimation step is possible only after the system has completed the identification of a road sign, which implies four stages: segmentation, detection, recognition and tracking. Specifically, in [7] the author describes all of these steps. 


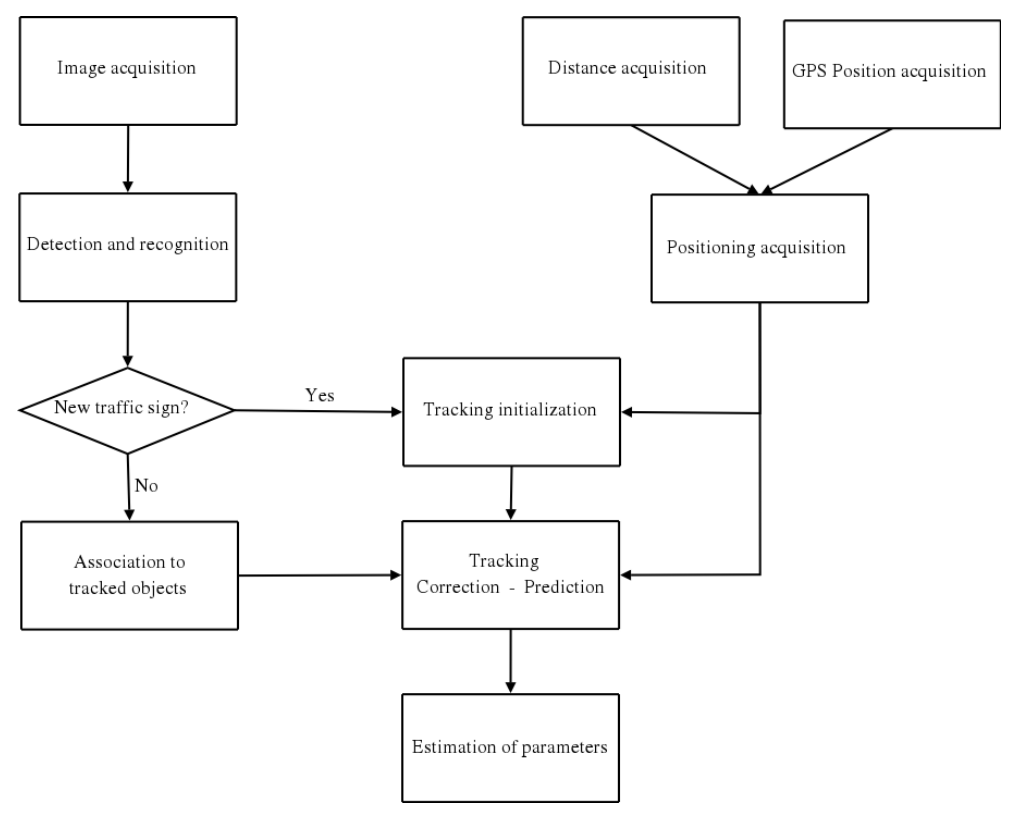

Fig. 2. System flow diagram

\section{Traffic Sign Parameters Estimation}

There are at least 350 of different road sign types. As we have described in the previous section, our system allows to detect and identify road signs in a sequence of video. This kind of systems require detailed knowledge of the sign, with the additional purpose of reducing false alarms and to make an inventory not only with the type of the found road sign during the route, but also with some parameters of each sign: height, dimensions, visibility distance, partial occlusion, GPS position, distance of separation between them. For an inventory system, these kind of data could be used to verify if the dimensions are standard, if two consecutive road signs are too closer, or if it exists any kind of partial occlusion which complicates that a driver can perceive the signal.

To obtain these estimations of parameters we have considered a pin-hole camera model, as shows Fig. 3. where they are known the following parameters: the focal length $(f)$, size of each unit cell $\left(u_{c h}\right.$ and $u_{c w}$, which are the height and width of a cell, respectively) in the Charge-Coupled Device (CCD) of the camera, the height of the camera $\left(h_{c}\right)$, the height of the middle point of a captured image $\left(h_{m}\right)$, and the distance between the camera plane and the middle point plane of a captured image $d_{m}$. Then, before starting to record a route, we have to complete a calibration step, which consists in to measure $h_{c}, h_{m}$ and $d_{m}$. We develop this step on a known area, where we can measure these distances without difficulties. 


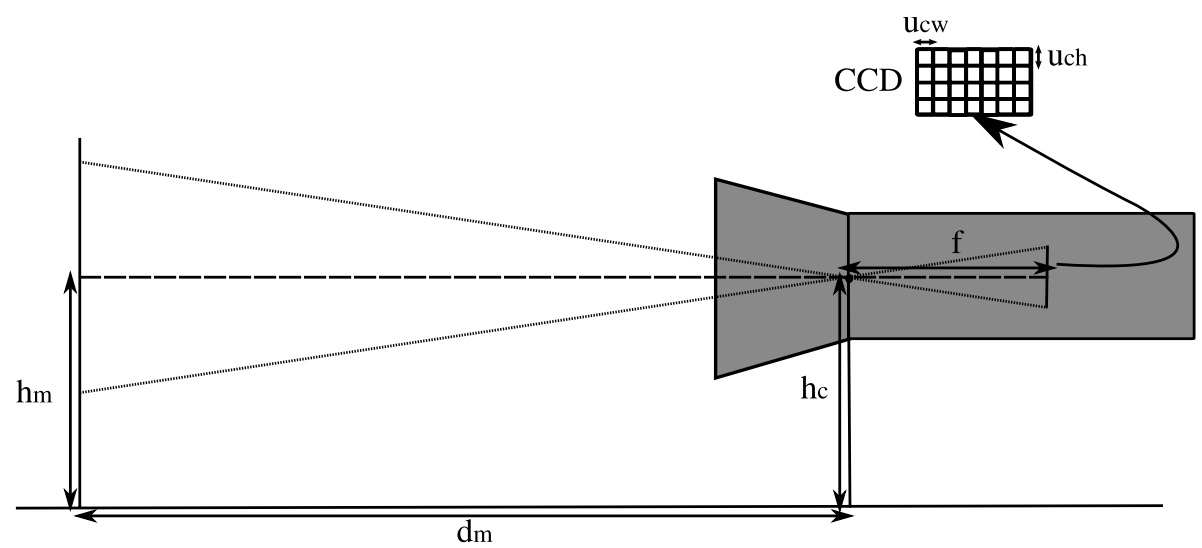

Fig. 3. Calibration of the system

\subsection{Height Estimation}

Our first objective is to estimate the height of the sign. Ideally, we could consider that the plane where is the road sign, and the plane where is projected the image in the camera, are parallel, but it does not happen in a real situation, because the sign or the camera, or both, can be inclined. We have to take into account this consideration to design a model which lets us to estimate the height of an object. Anyway, we must to define the model where camera and sign plane are parallel, and then, we will extend this explanation to the real situation.

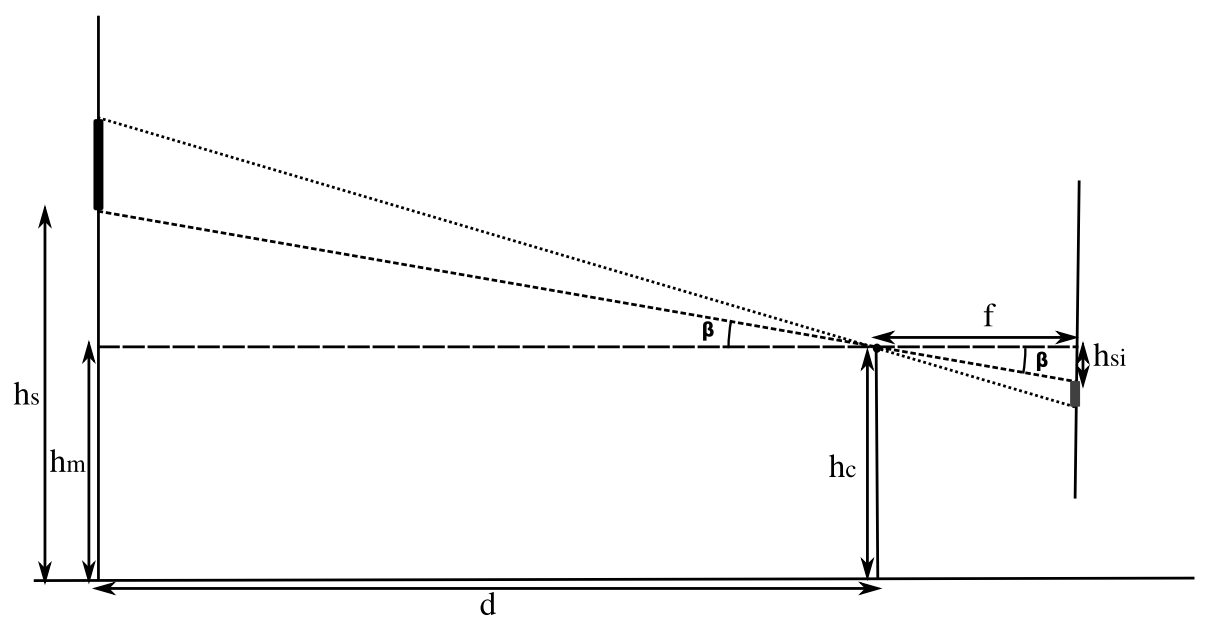

Fig. 4. Ideal situation where camera and road sign plane are parallel

Figure 4 shows the created model to estimate the height of a road sign. After the detection and recognition process, we have the position of the identified 
road sign in the image $h_{s i}=h_{s p} u_{c h}$, where $h_{s p}$ is the height in pixels and $u_{c h}$ is the height in $\mathrm{mm}$ of each pixel. The Positioning subsystem gives the covered distance between two images where the recognition subsystem had identified the same road sign. Then, knowing these data and that $h_{c}=h_{m}$ we can establish that

$$
\begin{gathered}
\tan (\beta)=\frac{h_{s i}}{f}, \\
\tan (\beta)=\frac{h_{s}-h_{c}}{d+x},
\end{gathered}
$$

where $x$ is the covered distance between two consecutive captured images where the signal had been recognized. For the second captured image we can define

$$
\begin{gathered}
\tan \left(\beta^{\prime}\right)=\frac{h_{s i}^{\prime}}{f}, \\
\tan \left(\beta^{\prime}\right)=\frac{h_{s}-h_{c}}{d} .
\end{gathered}
$$

From both pair of equations we can write

$$
\begin{aligned}
\frac{h_{s i}}{f} & =\frac{h_{s}-h_{c}}{d+x}, \\
\frac{h_{s i}^{\prime}}{f} & =\frac{h_{s}-h_{c}}{d} .
\end{aligned}
$$

These last two equations form the following system of equations

$$
\begin{gathered}
h_{s}-\frac{h_{s i}^{\prime}}{f} d=h_{c}, \\
h_{s}-\frac{h_{s i}}{f} d=h_{c}+\frac{h_{s i}}{f} x,
\end{gathered}
$$

where the unknowns are $h_{s}$ and $d$.

Our objective is to obtain the height of the road sign $h_{s}$ which is

$$
h_{s}=h_{c}+\frac{h_{s i}^{\prime} h_{s i} x}{f\left(h_{s i}^{\prime}-h_{s i}\right)} \text {. }
$$

We only have considered the situation in which the road sign is over the middle point of the captured image. Of course, we have two possible situations more. The traffic sign can be at the same height that the middle point, then $h_{s}=h_{m}$. The last situation is that $h_{s}<h_{m}$, and then we can write

$$
\begin{gathered}
\tan (\beta)=\frac{h_{s i}}{f}, \\
\tan (\beta)=\frac{h_{c}-h_{s}}{d+x},
\end{gathered}
$$




$$
\begin{gathered}
\tan \left(\beta^{\prime}\right)=\frac{h_{s i}^{\prime}}{f}, \\
\tan \left(\beta^{\prime}\right)=\frac{h_{c}-h_{s}}{d} .
\end{gathered}
$$

Now $h_{s}$ is defined as follows

$$
h_{s}=h_{c}-\frac{h_{s i}^{\prime} h_{s i} x}{f\left(h_{s i}^{\prime}-h_{s i}\right)} .
$$

In a real approach we must consider that the road sign and the camera plane are not parallel. Figure 5 shows this situation. Now, the angle $\alpha \neq 0$, and in a first stage of calibration we must measure this angle by

$$
\alpha=\arctan \left(\frac{h_{m}-h_{c}}{d_{m}}\right)
$$

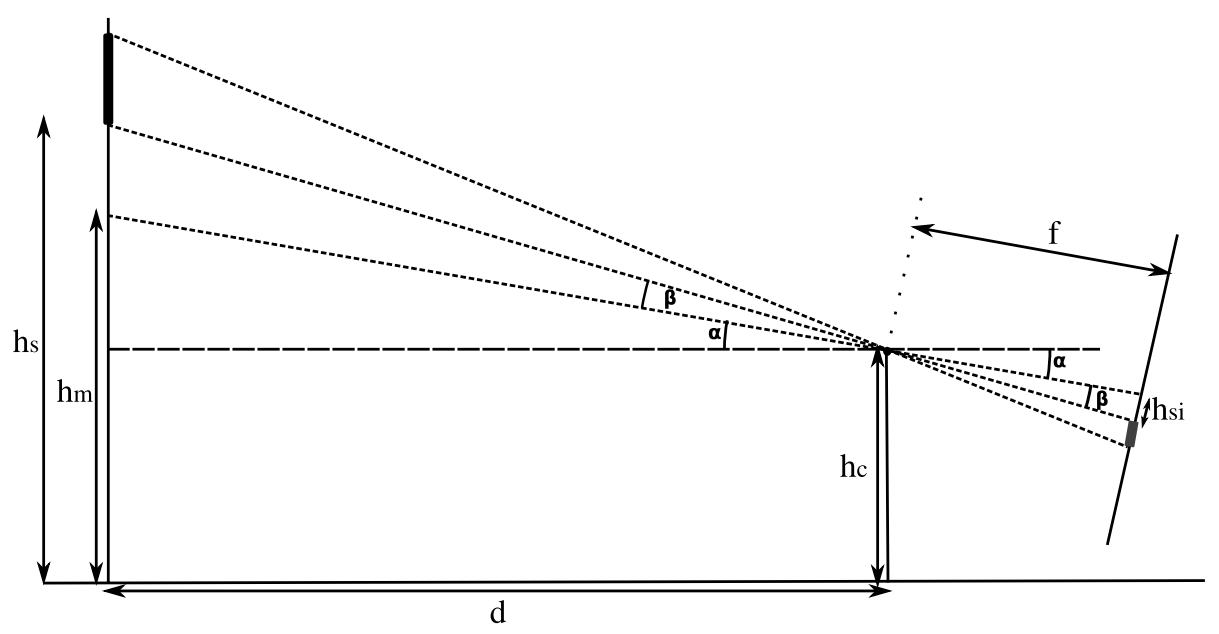

Fig. 5. Real situation where camera and road sign plane are not parallel

From this point, with $\alpha>0$, we can define

$$
\begin{gathered}
\beta=\arctan \left(\frac{h_{s i}}{f}\right), \\
\tan (\alpha+\beta)=\frac{h_{s}-h_{c}}{d+x} .
\end{gathered}
$$

For the next captured image we have

$$
\beta^{\prime}=\arctan \left(\frac{h_{s i}^{\prime}}{f}\right),
$$




$$
\tan \left(\alpha+\beta^{\prime}\right)=\frac{h_{s}-h_{c}}{d}
$$

Then, we have the following system of equations

$$
\begin{array}{r}
h_{s}-\tan (\alpha+\beta) d=h_{c}+\tan (\alpha+\beta) x, \\
h_{s}-\tan \left(\alpha+\beta^{\prime}\right) d=h_{c} .
\end{array}
$$

From this point, following the same manner of reasoning that in the ideal situation, we will have that $h_{s}$ must be estimated as follows

$$
h_{s}=h_{c}-\frac{x \tan (\alpha+\beta) \tan \left(\alpha+\beta^{\prime}\right)}{\tan (\alpha+\beta)-\tan \left(\alpha+\beta^{\prime}\right)},
$$

Also, if we consider the situation where $h_{s}<h_{m}$, then $h_{s}$ will be estimated as

$$
h_{s}=h_{c}-\frac{x \tan (\alpha-\beta) \tan \left(\alpha-\beta^{\prime}\right)}{\tan (\alpha-\beta)-\tan \left(\alpha-\beta^{\prime}\right)} .
$$

This mathematical study, about the trigonometric relationships between the real world and the projected image in a camera, lets us to estimate the height of a sign successfully.

\subsection{Dimensions Estimation}

To estimate the dimensions of a road sign is a task which is related with the type of the sign. We must know, before the dimensions estimation step, the answer to the following question: what kind of traffic sign do we have recognized?. With this information, we only have to apply the same algorithm that we have described in the previous section, but not only for the botton point of the sign. The detection and recognition subsystem gives us the vertexes of the rectangle where the road sign is confined. For a correct dimensions estimation, we have to estimate the height of the top and botton corner of this rectangle. Then, we can compute all the dimensions for every type of road sign.

\subsection{Visibility Distance Estimation}

We define the visibility distance as the distance where the road sign has been detected for the first time by our system. This parameter is crucial for and inventory system which wants to measure the level of safety of a road. To estimate this parameter, we have to take up again the approach to estimate the height of a road sign. Figure 4 shows the model for a ideal situation where road sign and camera plane are parallel. Equations (7) and (8) compose a systems of equations, where the unknowns are $d$ and $h_{s}$. We can work out the value of $d$, which is the visibility distance that we are searching.

$$
d=\frac{h_{s i} x}{h_{s i}^{\prime}-h_{s i}} .
$$


In a real situation we have to use the model presented in Fig. 5. Then, we can estimate the visibility distance as

$$
\begin{aligned}
& d=\frac{\tan (\alpha+\beta) x}{\tan \left(\alpha+\beta^{\prime}\right)-\tan (\alpha+\beta)} \text { if } h_{s}>h_{m} \\
& d=\frac{\tan (\alpha-\beta) x}{\tan \left(\alpha-\beta^{\prime}\right)-\tan (\alpha-\beta)} \text { if } h_{s}<h_{m}
\end{aligned}
$$

\subsection{Partial Occlusion Estimation}

Many times, traffic signs appear occluded by other objects like trees, vehicles, other road signs, etc. This traffic signs analysis system gives an estimation of partial occlusions, and to have this information of each sign results crucial for a complete inventory system which measures the level of maintenance of a road, including the safety. To estimate this parameter, we use the information provided by the tracking subsystem: if the same road sign is detected in non-consecutive frames, we can determine that a partial occlusion was happen.

\section{Results}

The current data of several traffic signs have been obtained for a sequence in a two ways road. For testing our approach we have measured the height and dimensions of some road signs, which are presented in Fig. 6

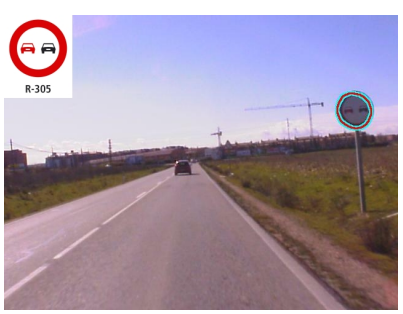

(a)

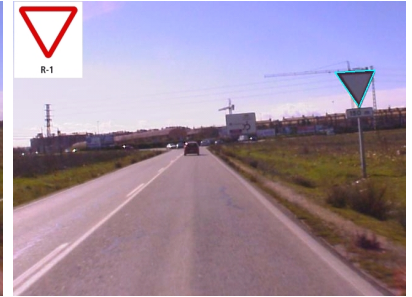

(b)

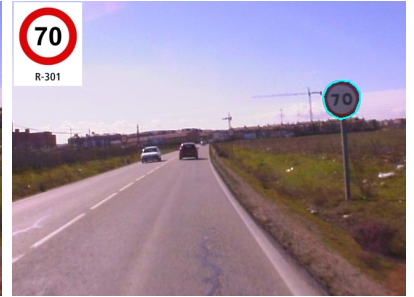

(c)

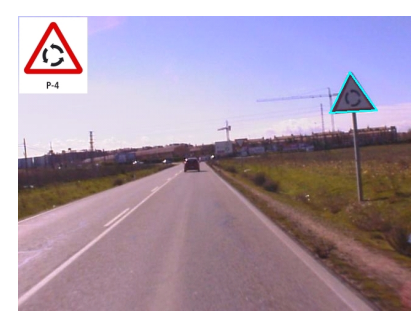

(d)

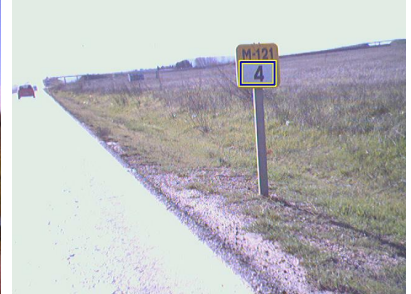

(e)

Fig. 6. Some detected and recognized road signs 
Table 1. Parameters estimation for some road signs

\begin{tabular}{cccc}
\hline Road Sign Real Height $(\mathrm{m})$ & Estimated Height $(\mathrm{m})$ & Error $(\%)$ \\
\hline a) & 1.95 & 1.90 & 2.5 \\
b) & 2.16 & 2.13 & 1.38 \\
c) & 1.84 & 1.81 & 1.6 \\
d) & 1.92 & 1.87 & 2.6 \\
\hline
\end{tabular}

Our test sequences of images have been recorded with two firewire cameras fixed onto the hood and the roof of the car. The captured images have a resolution of $640 \times 480$, and in a calibration step we have measured $h_{c}, h_{m}, f, d_{m}$ and $u_{c h}$. Figure 6 shows in image e) a milestone detected by our system, and Table 1 shows the estimated height for each type of sign and the error commited.

We can observe that the error obtained is not too high, and the parameter estimated is closer to the real value. The worse the measurement in the calibration step, the larger the error in the estimation. The most important cause of error in the estimation process is the measurement of the variable $x$, which indicates the covered distance between two captured images. The Positioning subsystem refreshes this variable each 1.6 meters, because this is the resolution of our DMD, then we do not have the exact value of this parameter. The presented mathematical procedure works well when all the variables are measured correctly, and the error obtained in this situation is about $0.8 \%$. But the error in this estimation can be caused by some other factors: lens distortion, an incorrect detection of the sign in the image. The dimensions of each sign are estimated following the same process, but for two point of the signal, as we have described in the previous sections.

Table 2 shows some estimated visibility distances.

Table 2. Visibility Distance Estimation

\begin{tabular}{cc}
\hline Road Sign Visibility Distance $(\mathrm{m})$ \\
\hline a) & 41.2 \\
b) & 37.6 \\
c) & 42.7 \\
d) & 21.6 \\
\hline
\end{tabular}

\section{Conclusions}

This paper describes a complete framework to estimate height, dimensions, partial occlusions and visibility distance of traffic signs. The integration of the recognition, tracking and positioning subsystems, allows to estimate these parameters automatically, following the mathematical approach presented from this work. 
Future lines of work will focus on estimating more attributes of each sign, such as deformation level, distance from the sign to the margin of the road, level of slope. On the other hand, we will work in a more complete system which will have calibrated cameras and a Positioning subsystems which improves the estimation of covered distance. Another future objective will be to complete the system with an stereo vision framework, in order to improve these estimations of parameters.

\section{Acknowledgment}

This work was supported be the project of the Ministerio de Educación y Ciencia of Spain number TEC2004/03511/TCM.

\section{References}

1. Maldonado, S., Lafuente, S., Gil, P., Gómez, H., López, F.: Road-sign detection and recognition based on support vector machines. IEEE Trans. on Intelligent Transportation Systems (2006)

2. de la Escalera, A., Armingol, J.M., Pastor, J.M., Rodríguez, F.J.: Visual sign information extraction and identification by deformable models for intelligent vehicles. IEEE Trans. on Intelligent Transportation Systems 15 (2004) 57-68

3. Fang, C., Chen, S.: Road sign detection and tracking. IEEE Trans. on Vehicular Technology 52 (2003) 1329-1341

4. Lafuente Arroyo, S., García Díaz, P., Acevedo Rodríguez, F., Gil Jiménez, P., Maldonado Bascón, S.: Traffic signs classification invariant to rotations using support vector machines. In: Proceedings of Advabced Concepts for Intelligent Vision Systems, Brussels, Belgium (2004)

5. de la Escalera, A., Moreno, L.E., Salichs, M.A., Armingol, J.M.: Road traffic sign detection and classification. IEEE Trans. on Industrial electronics 44 (1997) 848-859

6. Aoyagui, Y., Asakura: A study on traffic sign recognition in scene image using genetic alhorithms and neural networks. In: Proceedings IEEE Int. Conf. Industrial Electronics, Control and Instrumentation. Volume 3., Taipei, Taiwan (1996) 18381843

7. Lafuente-Arroyo, S., Maldonado-Bascón, S., Gil-Jiménez, P., Gómez-Moremo, H., López-Ferreras, F.: Road sign tracking with a predictive filter solution. In: Proc. of IEEE IECON, Paris, France (2006) 3314-3319 